\title{
Intraoperative three-dimensional transesophageal echocardiography for evaluating an unusual structure in the left ventricular outflow tract -a case report-
}

\author{
Tae-Yun Sung ${ }^{1}$, Won-Kyoung Kwon ${ }^{2}$, Dong-Ho Park ${ }^{1}$, Cheol-Hwan Park ${ }^{2}$, \\ and Tae-Yop Kim ${ }^{2}$ \\ Department of Anesthesiology and Pain Medicine, ${ }^{1}$ Konyang University Hospital, Konyang University College of \\ Medicine, Daejeon, ${ }^{2}$ Konkuk University Medical Center, Konkuk University School of Medicine, Seoul, Korea
}

\begin{abstract}
Intraoperative three-dimensional (3D) transesophageal echocardiography (TEE) facilitates an understanding of the complex cardiac pathology that is not fully delineated in a two-dimensional (2D) echocardiographic evaluation, and it suggests earlier and more precise surgical planning and intraoperative decision making. In the present case, the intraoperative 2D-TEE midesophageal long-axis view indicated a significant narrowing of the left ventricular outflow tract (LVOT) area by a band-like structure that vertically traversed the middle of the LVOT and connected to the anterior mitral leaflet base and the interventricular septum. However, additional 3D-TEE images of the LVOT and their cropped and rendered 2D images showed that web-like tissue, which presumably had grown around the patch closure from a previous atrioventricular septal defect, was obstructing the LVOT partially.
\end{abstract}

Key Words: Left ventricular outflow tract, Three dimensional echocardiography.

Intraoperative three-dimensional (3D) transesophageal echocardiography (TEE) facilitates an understanding of complex cardiac pathology that is not fully delineated by a two-dimensional (2D) echocardiographic evaluation, and it suggests earlier and

Corresponding author: Tae-Yop Kim, M.D., Ph.D.

Department of Anesthesiology and Pain Medicine, Konkuk University Medical Center, Konkuk University School of Medicine, 120-1, Neungdong-ro, Gwangjin-gu, Seoul 05030, Korea

Tel: 82-2-2030-5445, Fax: 82-2-2030-5449

E-mail: taeyop@gmail.com

ORCID: http://orcid.org/0000-0003-0806-8969

Received: September 15, 2014.

Revised: November 13, 2014.

Accepted: December 4, 2014.

Korean J Anesthesiol 2015 October 68(5): 505-508

http://dx.doi.org/10.4097/kjae.2015.68.5.505 more precise surgical planning and intraoperative decision making [1].

In the present case, 3D-TEE provided a clearer interpretation of the complex pathologic lesions that caused a partial obstruction in the left ventricular outflow tract (LVOT) area and the presence of mitral valve regurgitation (MR), which had not been delineated fully by preoperative $2 \mathrm{D}$ transthoracic echocardiography (TTE) or intraoperative $2 \mathrm{D}$-TEE evaluations.

\section{Case Report}

A 40-year-old male with intermittent chest pain and dyspnea on exertion underwent elective cardiopulmonary bypass (CPB) surgery for MR and partial obstruction of the LVOT diagnosed by $2 \mathrm{D}$-TTE. He underwent a patch-closure procedure for an atrioventricular septal defect (AVSD) 10 years previously. The intraoperative 2D-TEE midesophageal long-axis (ME-LAX) view

(c) This is an open-access article distributed under the terms of the Creative Commons Attribution Non-Commercial License (http://creativecommons.org/ licenses/by-nc/4.0/), which permits unrestricted non-commercial use, distribution, and reproduction in any medium, provided the original work is properly cited. 
showed a band-like structure vertically traversing the middle of the LVOT, connecting the anterior mitral leaflet (AML) base and the interventricular septum, and tethering the base of the AML anteriorly. These 2D-TEE images suggested that more than half

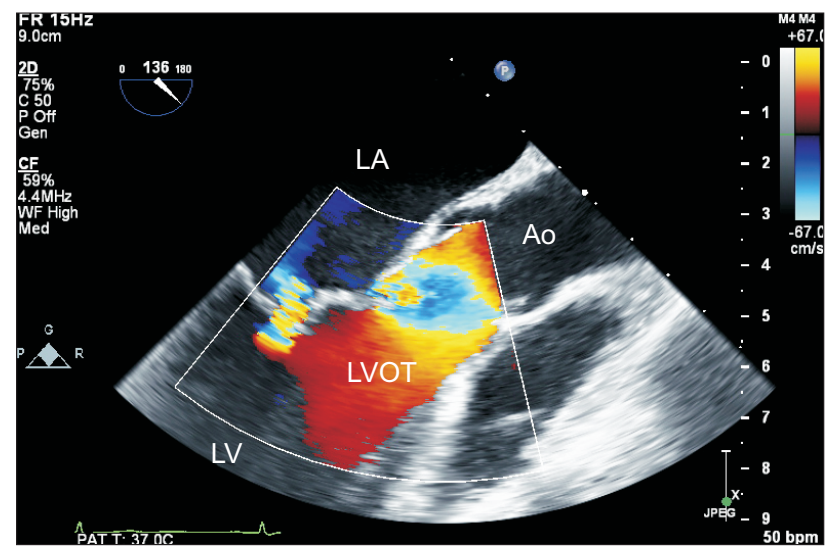

Fig. 1. Two-dimensional transesophageal echocardiography midesophageal long-axis view with color-flow Doppler. A mild eccentric MR jet is directed posteriorly. The mild MR was associated with tethering of the anterior mitral leaflet, but no hypertrophied region or bulging in the interventricular septum was detected in the LVOT area. The echogenic masses vertically traversed the entire LVOT area, suggesting compromised LVOT patency. LA: left atrium, LV: left ventricle, LVOT: left ventricular outflow tract, Ao: aorta, MR: mitral regurgitation. the LVOT area was obstructed by this band-like structure (Fig. 1 and Video 1). However, additional 3D-TEE (X7-2t $\mathrm{t}^{\mathrm{TM}}$ and $\mathrm{iE} 33^{\mathrm{TM}}$; Philips Healthcare, Bothell, WA, USA) images consisting of an en face view of the LVOT (Video 2) and cropped and rendered $2 \mathrm{D}$ images provided more comprehensive information to understand the pathophysiology and anatomic relationship of LVOT structures, including the AML and interventricular septum; this information was quite different from that provided by $2 \mathrm{D}$ echocardiography. The web-like tissue in the parietal band region of the interventricular septum was asymmetrically obstructing the right corner (posteromedial aspect) of the LVOT. However, the peak velocity and peak pressure gradient of the LVOT were about $80 \mathrm{~cm} / \mathrm{s}$ and $2.6 \mathrm{mmHg}$ in pulsed-wave Doppler analyses in the deep transgastric view. The degree of obstruction confirmed in the en face view of the LVOT seen from the left ventricle (LV) perspective showed that the band-like structure was partially tethering the base of the AML, but it was not traversing the center of the LVOT; the narrowest part of the LVOT and subaortic region measured as approximately $2.3 \mathrm{~cm}^{2}$ using a multiple cross-sectional cropping process in the 3D-TEE software (Q$\mathrm{lab}^{\mathrm{TM}}, \mathrm{MVQ}^{\mathrm{TM}}$ and i-slice ${ }^{\mathrm{TM}}$, Philips, San Jose, CA, USA) (Fig. 2 and Video 3).

After initiating $\mathrm{CPB}$, the direct surgical view under surgical exposure of a left atriotomy confirmed the folded and slightly

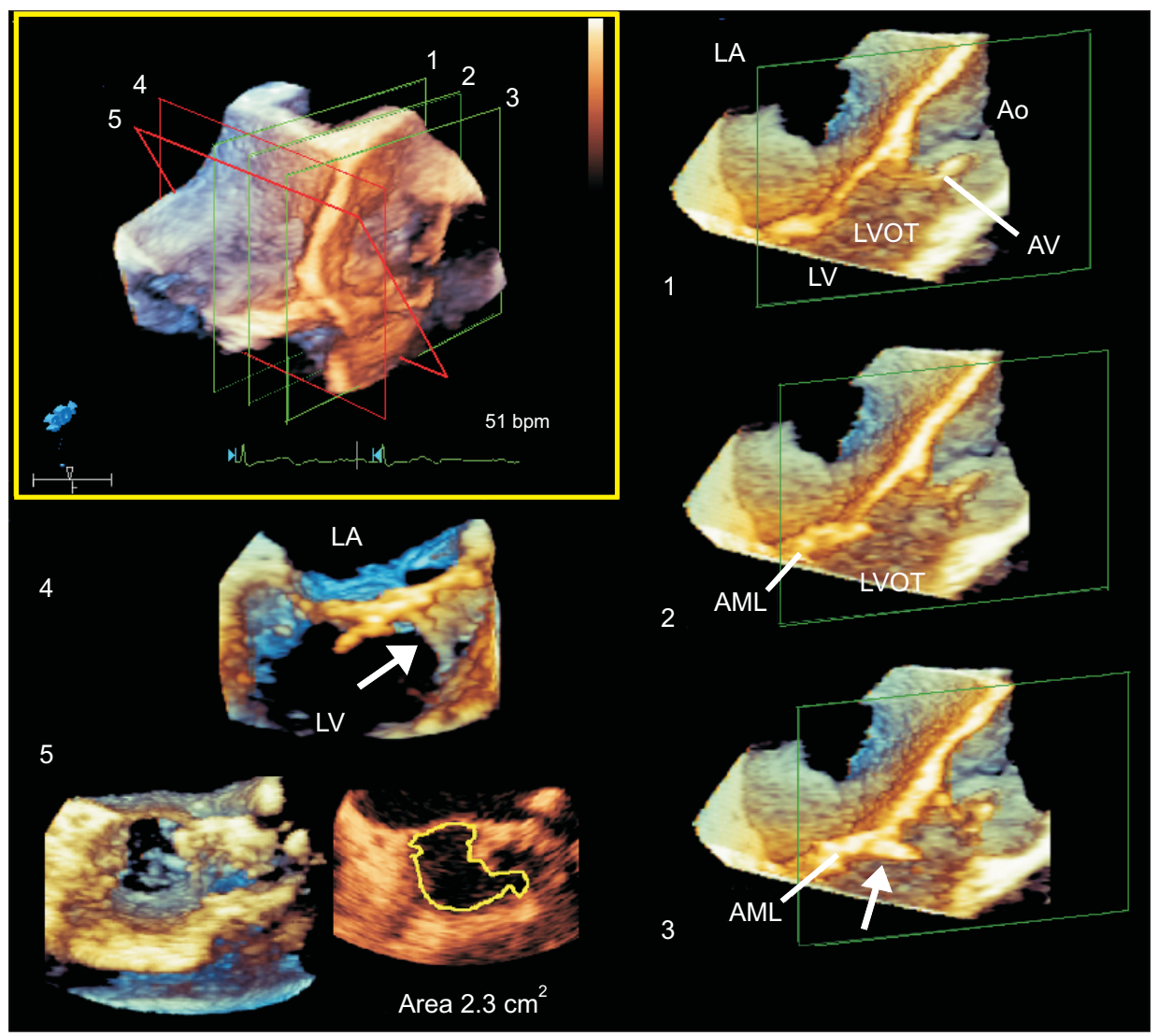

Fig. 2. Multiple three-dimensional (3D) cropped and rendered coronal images parallel (1-3) and perpendicular (4) to a single 3D zoom-volume image of the LVOT (left upper). 3D en face image of the LVOT seen from the LV perspective reveals a band-like structure (arrows in 3 and 4) occupying the septal corner of the LVOT, but not significantly obstructing the LVOT (5); the narrowest 3D-planimetric LVOT area, which was measured on multiple cross-sectional images using 3D-transesophageal echocardiography software, was $2.32 \mathrm{~cm}^{2}$. LA: left atrium, LV: left ventricle, LVOT: left ventricular outflow tract, Ao: aorta, AV: aortic valve, AML: anterior mitral leaflet. 

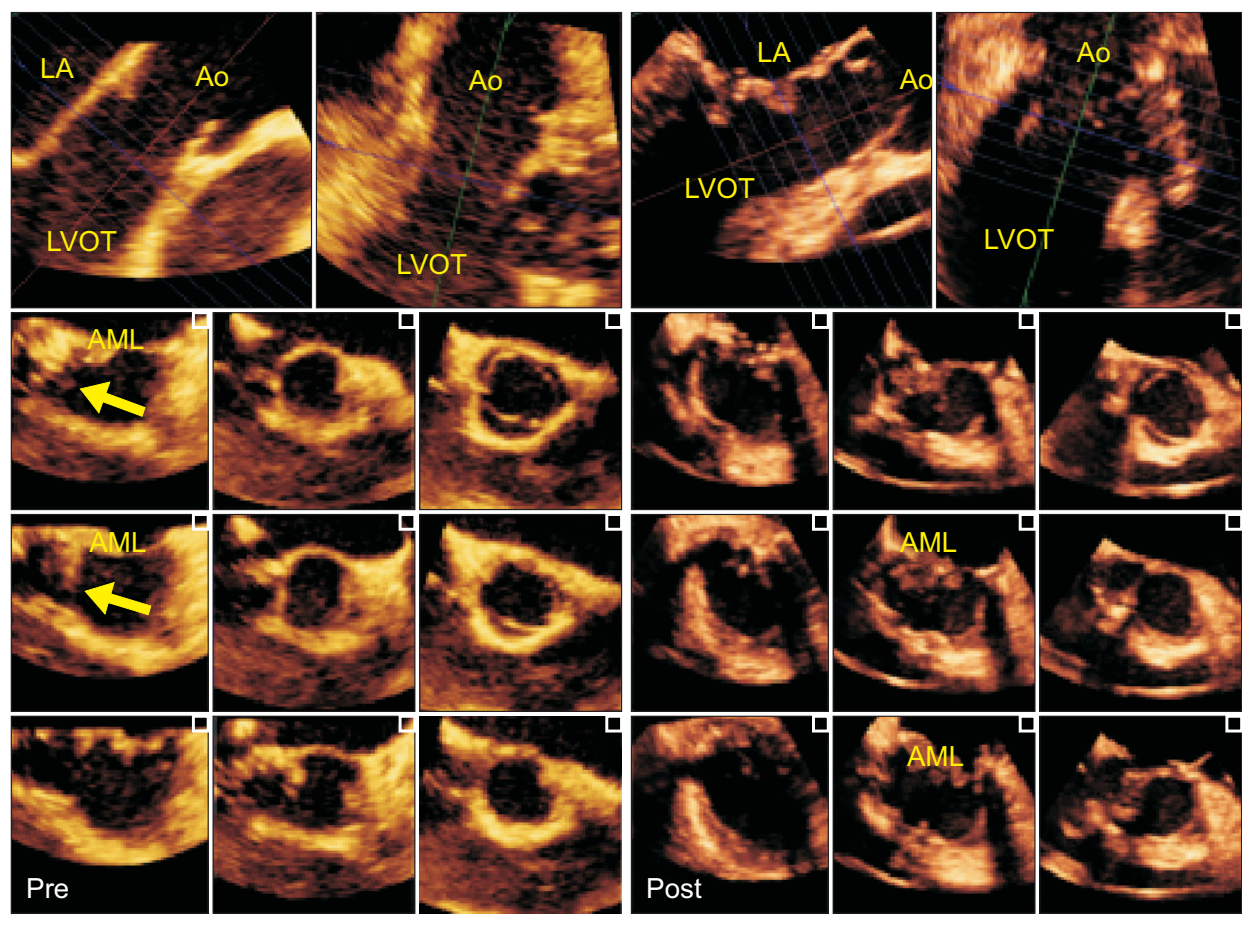

Fig. 3. Multiple two-dimensional views of the LVOT derived from three-dimensional volume data by using a software producing multi-slice 2D images (i-slice ${ }^{\mathrm{TM}}$, Philips Healthcare, Bothell, WA, USA) in before and after cardiopulmonary bypass. The folded and slightly bulged web-like tissue (yellow arrow) was partially obstructing the posteromedial aspect of the LVOT (left). After resecting the web-like tissue, multiple LVOT images revealed a fully widened LVOT, resolution of the AML tethering, and incomplete MV coaptation producing mild MR (right). LA: left atrium, LVOT: left ventricular outflow tract, Ao: aorta, AML: anterior mitral leaflet, Pre: before surgical procedure, Post: after surgical procedure. bulged web-like tissue, which partially obstructed the posteromedial aspect of the LVOT, as shown in the 3D-TEE image. The tissue had grown around the patch closure used for a previous AVSD. The redundant patch applied during the previous AVSD closure was removed and replaced with a new pericardial patch, and the abnormally grown web-like tissue was resected (Fig. 3). As a lifting annuloplasty (augmentation of the posterior leaflet), plication of the posterior annulus was performed by suturing a flexible ready-to-use strip (Mitra-Lift ${ }^{\mathrm{TM}}$; Sciencity, Seoul, Korea) onto the atrial wall above the posterior annulus between both commissures. After the surgical procedure, the peak pressure gradient of the LVOT was about $2.2 \mathrm{mmHg}$. The $2 \mathrm{D}$ - and 3DTEE images after weaning from CPB revealed a fully widened LVOT $\left(2.8 \mathrm{~cm}^{2}\right.$ on $3 \mathrm{D}$ planimetry), resolution of the AML tethering, and incomplete MV coaptation producing mild MR (Fig. 3 and Video 4).

\section{Discussion}

LVOT obstruction requiring reoperation can rarely occur after AVSD correction. In case of LVOT obstruction after AVSD correction, in $83 \%$, it was diagnosed 2 to 14 years postoperatively. Thus, postoperative follow-up echocardiography is important to early detection of LVOT obstruction resulted from various causes [2]. In the present case, web-like tissue grew abnormally around a patch that had been used for a previous AVSD closure. It occupied the corner of the LVOT, was tethered to the base of the AML, and partially contributed to the MR. However, pre- operative and intraoperative 2D echocardiographic evaluations were insufficient for providing a comprehensive understanding of the associated cardiac pathology. The degree of obstruction determined by $2 \mathrm{D}$ echocardiography suggested that the band/ web-like structure had completely obstructed the entire LVOT. These 2D findings were not matched well to the severity of the obstruction indicated by the Doppler velocity profile, the patient's symptoms, or the direct surgical findings.

Conventional 2D echocardiographic imaging provides limited information in a single slice tomographic image and requires the examiner to mentally reconstruct through an understanding of the structural relationships of 3D cardiac structures. The authors concluded that the 2D ME-LAX image was not appropriately aligned in the center of the LVOT, as it deviated slightly to the posteromedial side. Various 2D images taken in various tomographic planes and their integration are necessary for a comprehensive understanding of complex cardiac pathology, as in the present case. However, since the image planes of TEE tomography are limited and parallel to the orientation of the cardiac axis, it is difficult to align the image planes to accurately and appropriately visualize the tubular and elliptical LVOT structure; even use of multiple 2D-TEE images is occasionally insufficient for a thorough evaluation of complex cardiac pathology [3].

In contrast, 3D-TEE showed that additional clinical efficacy by making a comprehensive spatial image from $2 \mathrm{D}$ images [4]. The ability of 3D-TEE to produce volume images (dataset) of objective cardiac structures enables dimensionless cardiac imaging in the true anatomic perspective by freely modifying 
the volume image through rotation, cropping, and rendering, which facilitates decision making [5]. It could be possible for surgeons to confirm the LVOT pathology, seen from one side of the LV or aorta perspective, through the direct inspection after atriotomy or aortotomy. However, 3D-TEE images enables the anesthesiologist to observe the both sides that LV and aortic valve perspective by rotating the volume images. In the present case, a single $3 \mathrm{D}$-TEE en face high quality image seen from the LV perspective provided precise LVOT anatomy, comparable or superior to that seen by a surgeon during a surgical procedure. This image enabled a more accurate and rapid evaluation of the pathology, as shown in previous studies [3-8]. These precise and prompt pre-procedural evaluation may also be facilitate surgical procedure by preventing the potential intraoperative delay due to identification of the discrepancy between the pr-procedural diagnosis and surgical inspection [8].

Real-time 3D imaging also benefits surgeons by facilitating faster and more accurate communication for decision making; just "seeing" a single 3D image displayed on the TEE monitor enabled their comprehensive understanding. Gripari et al. [1] demonstrated that a surgeon can detect the cardiac pathology on 3D-TEE images more easily and accurately compared with corresponding $2 \mathrm{D}$ images.

In conclusion, the present case showed that intraoperative 3D-TEE enabled a more rapid and accurate assessment of complex pathology resulting in an LVOT obstruction with MR. Although intraoperative 2D-TEE imaging is sufficient in most clinical situations, the information obtained from additional 3DTEE imaging can be of enormous benefit for surgical planning and post-surgical follow up.

\section{References}

1. Gripari P, Tamborini G, Barbier P, Maltagliati AC, Galli CA, Muratori M, et al. Real-time three-dimensional transesophageal echocardiography: a new intraoperative feasible and useful technology in cardiac surgery. Int J Cardiovasc Imaging 2010; 26: 651-60.

2. Cho JY. Reoperations for Left Atrioventricular Valve Regurgitation and Left Ventricular Outflow Tract Obstruction After Atrioventricular Septal Defect Correction. J Korean Pediatr Cardiol Soc 2005; 9: 212-8.

3. Saitoh T, Shiota M, Izumo M, Gurudevan SV, Tolstrup K, Siegel RJ, et al. Comparison of left ventricular outflow geometry and aortic valve area in patients with aortic stenosis by 2-dimensional versus 3-dimensional echocardiography. Am J Cardiol 2012; 109: 1626-31.

4. Kim J, Yu GY, Seok J, Oh CS, Kim SH, Kim TY. Imaging of coronary artery fistulae by using intraoperative three-dimensional transesophageal echocardiography. Anesth Analg 2014; 118: 721-4.

5. Vegas A, Meineri M. Three-dimensional transesophageal echocardiography is a major advance for intraoperative clinical management of patients undergoing cardiac surgery: a core review. Anesth Analg 2010; 110: 1548-73.

6. Kwak J, Andrawes M, Garvin S, D’Ambra M. 3D transesophageal echocardiography: a review of recent literature 2007-2009. Curr Opin Anaesthesiol 2010; 23: 80-8.

7. Furukawa A, Abe Y, Tanaka C, Ito K, Tabuchi I, Osawa K, et al. Comparison of two-dimensional and real-time three-dimensional transesophageal echocardiography in the assessment of aortic valve area. J Cardiol 2012; 59: 337-43.

8. Jung HJ, Yu GY, Seok JH, Oh C, Kim SH, Yoon TG, et al. Usefulness of intraoperative real-time three-dimensional transesophageal echocardiography for pre-procedural evaluation of mitral valve cleft: a case report. Korean J Anesthesiol 2014; 66: 75-9. 